\title{
Treatment recommendations for psoriatic arthritis
}

\author{
C T Ritchlin, ${ }^{1}$ A Kavanaugh, ${ }^{2}$ D D Gladman, ${ }^{3}$ P J Mease, ${ }^{4}$ P Helliwell, ${ }^{5}$ W-H Boehncke, ${ }^{6}$ \\ $\mathrm{K}$ de Vlam, ${ }^{7}$ D Fiorentino, ${ }^{8} 0$ FitzGerald, ${ }^{9} \mathrm{~A}$ B Gottlieb, ${ }^{10} \mathrm{~N} \mathrm{~J} \mathrm{McHugh,},{ }^{11} \mathrm{P}$ Nash, ${ }^{12}$ \\ A A Qureshi, ${ }^{13}$ E R Soriano, ${ }^{14}$ W J Taylor, ${ }^{15}$ for the Group for Research and \\ Assessment of Psoriasis and Psoriatic Arthritis (GRAPPA)
}

\section{- Additional data}

(supplementary appendix 1) are published online only at http:// ard.bmj.com/content/vol68/ issueg

${ }^{1}$ University of Rochester Medical Center, Rochester, New York, USA; ${ }^{2}$ University of California at San Diego, San Diego, California, USA

${ }^{3}$ University of Toronto, Toronto, Ontario, Canada; ${ }^{4}$ Seattle Rheumatology Associates, Seattle, Washington, USA

${ }^{5}$ Academic Unit of

Musculoskeletal and

Rehabilitation Medicine,

University of Leeds, Leeds, UK;

${ }^{6}$ Johann Wolfgang Goethe

University, Frankfurt, Germany;

${ }^{7}$ University Hospitals Leuven,

Leuven, Belgium; ${ }^{8}$ Stanford

University, Stanford, California,

USA; ${ }^{9}$ St. Vincent's University

Hospital, Dublin, Ireland; ${ }^{10}$ Tufts

Medical Center, Boston,

Massachusetts, USA; ${ }^{11}$ Royal

National Hospital for Rheumatic

Diseases, Bath, UK; ${ }^{12}$ University

of Queensland, Cotton Tree,

Queensland, Australia;

${ }^{13}$ Harvard Medical School,

Boston, Massachusetts, USA;

${ }^{14}$ Hospital Italiano de Buenos

Aires, Buenos Aires, Argentina

${ }^{15}$ University of Otago/

Wellington, Wellington, New

Zealand

Correspondence to:

C T Ritchlin, Clinical Immunology

Research Center, University of

Rochester Medical Center, 601

Elmwood Avenue, Box 695 .

Rochester, New York 14642,

USA; christopher ritchlin@

urmc.rochester.edu

Accepted 26 September 2008

Published Online First

24 October 2008

\begin{abstract}
Objective: To develop comprehensive recommendations for the treatment of the various clinical manifestations of psoriatic arthritis (PsA) based on evidence obtained from a systematic review of the literature and from consensus opinion.
\end{abstract}

Methods: Formal literature reviews of treatment for the most significant discrete clinical manifestations of PsA (skin and nails, peripheral arthritis, axial disease, dactylitis and enthesitis) were performed and published by members of the Group for Research and Assessment of Psoriasis and Psoriatic Arthritis (GRAPPA). Treatment recommendations were drafted for each of the clinical manifestations by rheumatologists, dermatologists and PsA patients based on the literature reviews and consensus opinion. The level of agreement for the individual treatment recommendations among GRAPPA members was assessed with an online questionnaire. Results: Treatment recommendations were developed for peripheral arthritis, axial disease, psoriasis, nail disease, dactylitis and enthesitis in the setting of PsA. In rotal, 19 recommendations were drafted, and over 80\% agreement was obtained on 16 of them. In addition, a grid that factors disease severity into each of the different disease manifestations was developed to help the clinician with treatment decisions for the individual patient from an evidenced-based perspective.

Conclusions: Treatment recommendations for the cardinal physical manifestations of PsA were developed based on a literature review and consensus between rheumatologists and dermatologists. In addition, a grid was established to assist in therapeutic reasoning and decision making for individual patients. It is anticipated that periodic updates will take place using this framework as new data become available.

The articular and dermatological manifestations associated with psoriatic arthritis (PsA) are remarkably heterogeneous in the extent and type of tissue involvement. Patients with PsA, a chronic systemic inflammatory disorder, may develop not only peripheral arthritis but also axial disease, dactylitis, enthesitis and skin and nail psoriasis, with consequent adverse impact on function and quality of life (QoL). ${ }^{12}$ Heterogeneity is observed not only in disease manifestations but also in severity and course, which can vary from very mild psoriasis or enthesitis to widespread psoriatic plaques, disfiguring nail disease and severe joint inflammation with destruction that can result in disability and increased mortality. ${ }^{3}$ Moreover, comorbidities associated with psoriasis such as the metabolic syndrome can contribute to damage in multiple end-organs and often leads to markedly impaired QoL as well as early mortality. ${ }^{5-7}$

Recent progress in understanding the immunopathogenesis of PsA has been accompanied by treatment advances that have accelerated rapidly over the last decade. ${ }^{8}$ Despite these advances, therapeutic decisions for an individual patient with PsA can be challenging due to the diversity of clinical characteristics and the simultaneous involvement of multiple different tissues, often with varying degrees of severity. To address the need for evidence-based treatment recommendations and assist the practitioner, members of the Group for Research and Assessment of Psoriasis and Psoriatic Arthritis (GRAPPA) published systematic reviews of the literature to identify the best available evidence regarding treatment of the various manifestations of PsA. ${ }^{19}$ Herein, we present treatment recommendations that were formulated by rheumatologists and dermatologists in GRAPPA in conjunction with PsA patients, based on evidence from these systematic reviews and consensus opinion. These recommendations were developed to provide the best care for patients with PsA, regardless of economic or political considerations.

\section{METHODS}

The target audience for these treatment recommendations is all clinicians who care for PsA patients. First, formal literature reviews were performed by members of GRAPPA. To capture data regarding the varied areas of involvement characteristic of PsA, articles were selected that provided evidence supporting the treatment of peripheral arthritis, spinal disease, skin and nail disease, enthesitis and dactylitis in the setting of PsA (fig 1). These articles were reviewed and graded, and the results have been published. ${ }^{10-16}$ The evidence was graded using the approach of the Institute of Medicine. ${ }^{17}$ Wherever possible, effect sizes were calculated to quantify the extent of efficacy or toxicity. Effect size is the mean difference in effect between treatment and control, divided by the standard deviation of the difference. ${ }^{18}$ Effect sizes of 0.2 or less are considered small and unimportant in terms of efficacy, whereas effect sizes greater than 0.8 are considered large and suggest high efficacy.

Reviewers graded the evidence and treatment recommendations for PsA in accordance with recommendations from the Agency for Health Care Policy Research (AHCPR), as shown in table 1.

To address the nuanced and complex application of the results of these studies to the heterogeneous 
Figure 1 Group for Research and Assessment of Psoriasis and Psoriatic Arthritis (GRAPPA) treatment guidelines for psoriatic arthritis, categorised by disease characteristics and distinct organ involvement. Anti-TNF, anti-tumour necrosis factor; CsA, ciclosporin $A$; DMARD, disease-modifying antirheumatic drug; IA, intra-articular; LEF, leflunomide; MTX, methotrexate; NSAID, non-steroidal anti-inflammatory drug; $\mathrm{PT}$, physiotherapy; PUVA, psoralenultraviolet light $A$; $S S Z$, sulfasalazine; UVB, ultraviolet light B. Reproduced with permission from Kavanaugh et al.'

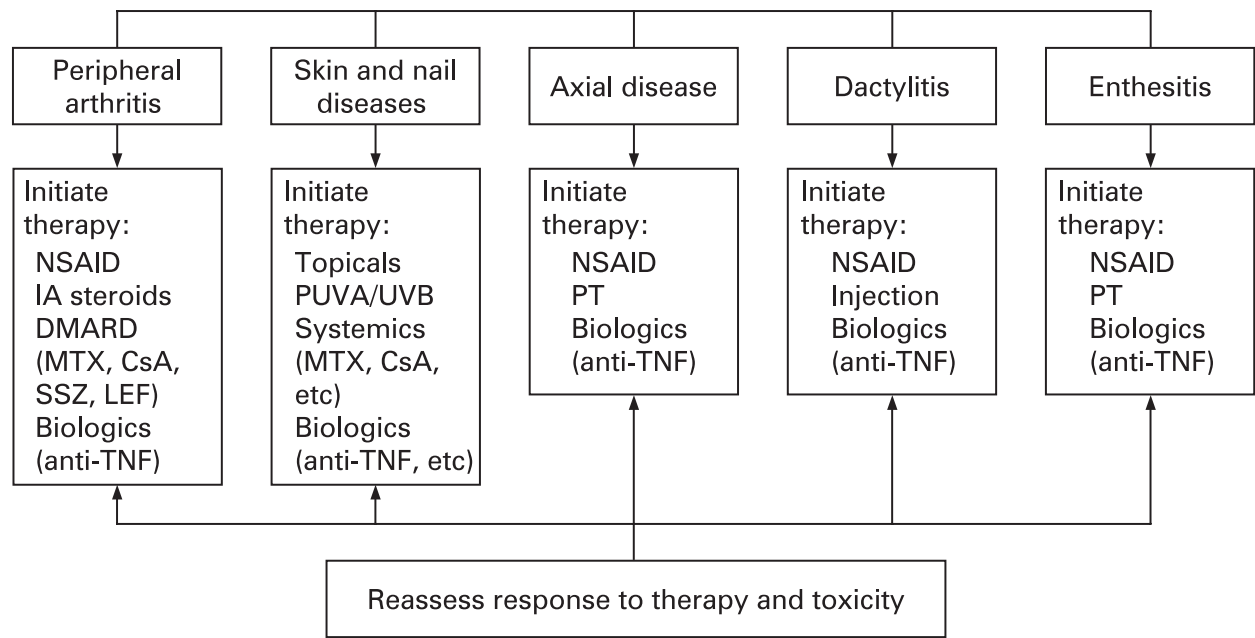

situations that arise in the clinic, focus groups were assembled, comprising experts in rheumatology and dermatology with specific experience in the care of PsA and psoriasis and patients with PsA. Next, subcommittees were formed for each of the five domains, and recommendations were drafted based on evidence and consensus between rheumatologists, dermatologists and patients for psoriasis, peripheral arthritis, axial disease, dactylitis and enthesitis. Each of these subcommittees developed a definition of mild, moderate, or severe for their individual domains. Finally, the subcommittee chairs met with AK and CTR (authors) to refine the recommendations (mild-moderatesevere categories) in each domain. The recommendations for each domain and the grid with the three categories were voted on by the entire membership of GRAPPA. Note that the grid was designed as a tool to assist in making treatment decisions for individual patients and as such is largely based on expertise. Data that became available after the systematic reviews were published were also considered by the various subcommittees.

A total of 19 items covering the diagnosis, assessment and treatment of the 5 PsA clinical manifestations were submitted for a vote using a web-based interface (Survey Monkey; http:// www.surveymonkey.com/). Each item was rated on a 5-point scale $(1=$ strongly agree to $5=$ strongly disagree). The Disagreement Index (DI) was derived from the 30th and 70th percentile of the respondents' ratings, adjusted for symmetry between the central point of the interpercentile range and the mid-point of the rating scale. The adjustment factor was derived from experimental work that compared different definitions of what constituted "disagreement" among panels of various sizes. The number of respondents, percentage respondents in the 30 th and 70 th percentile, percentage respondents in category 1 or 2 , and the mean and standard deviation were calculated.

\section{RESULTS}

The results of the survey are shown in the supplementary material. In all, 70 rheumatologists and dermatologists responded to the questionnaire. For 16 of the 19 items, 80\% of the respondents agreed or strongly agreed. The three areas where agreement was not as strong included the use of the Bath Ankylosing Spondylitis Disability Activity Index (BASDAI) to measure axial disease activity over time $(75.7 \%)$ and to assess axial treatment response $(78.7 \%)$ and the algorithm for the treatment of psoriasis (69.2\%). The strength of each recommendation (grades A-D) is included.

\section{Peripheral arthritis}

Diagnosis and assessment

Diagnosis of PsA should follow the CASPAR (for Classification Criteria for Psoriatic Arthritis) criteria. ${ }^{19}$ We consider inflammation to include such features as pain involving joints, spine and/ or enthuses associated with erythaema, warmth and swelling; prominent morning and rest stiffness.

Table 1 Grading of evidence sources and recommendations

\begin{tabular}{ll}
\hline Evidence or recommendation & Grade \\
\hline Evidence source as recommended by the Agency for Health Care Policy Research (AHCPR): & \\
Meta-analysis of randomised controlled trials (RCT) & $1 \mathrm{a}$ \\
One or more RCT & $1 \mathrm{~b}$ \\
One or more controlled trials (without randomisation) & $2 \mathrm{a}$ \\
Other well designed studies (quasiexperimental) & $2 \mathrm{~b}$ \\
Non-experimental studies (descriptive studies such as comparative or correlation studies, or case-control studies) & 3 \\
Expert committee opinions, clinical experience & 4 \\
Preliminary recommendations for treatment of psoriatic arthritis (using the best available evidence extracted from & \\
published literature): & $\mathrm{A}$ \\
Category 1 evidence & $\mathrm{B}$ \\
Category 2 evidence, or extrapolation from category 1 evidence & $\mathrm{C}$ \\
Category 3 evidence, or extrapolation from category 1 or 2 evidence & $\mathrm{D}$ \\
Category 4 evidence or extrapolation from category 2 or 3 evidence & \\
\hline
\end{tabular}


Preferably, diagnosis of psoriasis should be confirmed by a dermatologist and inflammatory musculoskeletal disease by a rheumatologist, or either one by an appropriately qualified health professional.

Baseline evaluations of PsA should include the following domains (consensus on core set of domains for psoriatic arthritis assessment established at the eighth Outcome Measures in Rheumatology (OMERACT 8) conference, 2006). ${ }^{20}$

- Peripheral joint assessment (68 joints for tenderness; 66 joints for swelling).

- Pain (patient-reported on a visual analogue or category rating scale).

- Patient global assessment of disease activity.

- Physical function (eg, as measured by the Health Assessment Questionnaire (HAQ)).

- Health-related QoL, as assessed by a general measure (eg, Short Form 36 (SF-36)) or a PsA-specific measure (eg, Psoriatic Arthritis QoL (PsAQoL)).

- Fatigue, measured by patient self-report or a general instrument (eg, Functional Assessment of Chronic Illness Therapy (FACIT)).

- Acute phase reactants (eg, C-reactive protein (CRP) or erythrocyte sedimentation rate (ESR)).

Radiographic assessment is encouraged according to clinical manifestation and doctor discretionary judgment.

Factors associated with a poor prognosis related to the progression of peripheral joint disease and damage in patients with PsA include: the number of actively inflamed joints (ie, polyarticular disease, as opposed to monoarticular disease); elevated ESR; failure of previous medication trials; the presence of damage, either clinically or on $x$ ray; ${ }^{21}$ a loss of function as assessed by HAQ; and diminished QoL as assessed by SF-36, Dermatology Life Quality Index (DLOI), or PsAQoL.

A patient should be considered a treatment failure when in spite of therapy for a length of time appropriate to the pharmacokinetic/pharmacodynamic profile of the individual agent at an appropriate dose, the patient fails to demonstrate acceptable clinical improvement. Response to treatment of peripheral arthritis in patients with PsA may be assessed using criteria initially developed for rheumatoid arthritis (RA), such as the 28-joint Disease Activity Score (DAS28, shown to be reliable and discriminative in PsA, even though it uses only 28 joints) and the European League Against Rheumatism (EULAR) response criteria, which categorise levels of disease and changes to assess response. The American College of Rheumatology (ACR) percentage response criteria (eg, ACR20/50/70) may also be used in PsA. ${ }^{22}$ In a recent analysis of PsA and RA outpatient cohorts, ${ }^{23}$ the utility of the DAS28 for PsA was questioned with regard to its applicability in settings outside of clinical trials, where patients receive therapies of varying efficacy. Response may also be considered inadequate if there is evidence of progression of joint damage on radiographs.

\section{Treatment}

Treatment recommendations for peripheral arthritis include non-steroidal anti-inflammatory drugs (NSAIDs), intra-articular glucocorticoid injections, disease-modifying antirheumatic drugs (DMARDs) and TNF inhibitors (see table 2).

Systemic corticosteroids are not typically recommended in the treatment of psoriasis and are only advisable in discrete circumstances and not for chronic use, due to the potential to cause post-steroid psoriasis flare and other adverse effects (D).
Gold salts, chloroquine and hydroxychloroquine also are not recommended for use in PsA.

DMARDs have the potential to reduce or prevent joint damage and preserve joint integrity and function (although none have been shown to do this in PsA). Many factors influence the choice of DMARD for the individual patient: its relative efficacy, convenience of administration, requirements of the monitoring program, costs of the medication and monitoring (including doctor visits and laboratory costs), time until expected benefit and the frequency and potential seriousness of adverse reactions. Input from a rheumatologist is often essential when initiating DMARD therapy.

A patient should be considered a DMARD failure if at least one DMARD has been failed individually or in combination in an adequate therapeutic trial, defined as treatment for $\geqslant 3$ months, of which $\geqslant 2$ months is at standard target dose (unless significant intolerance or toxicity limits the dose). Intolerance/toxicity is defined as treatment for $<2$ months, where treatment is withdrawn because of drug intolerance or toxicity. When treatment is withdrawn because of intolerance or toxicity after $>2$ months therapy, at least 2 months should have been at therapeutic doses.

Although there is no evidence for the use of combination therapy, a combination of two or more agents could be used in those patients who fail to respond to a single agent, or who present joint damage progression in spite of treatment.

\section{Axial disease}

\section{Diagnosis and assessment (based on ankylosing spondylitis (AS)} criteria)

Diagnosis of axial disease should be based on the presence of two of three of the following criteria:

- Inflammatory back pain (features including onset age $<45$ years, symptoms $>3$ months, morning stiffness $>30 \mathrm{~min}$, insidious onset, improved with exercise, alternating buttock pain).

- Limitation of motion of cervical, thoracic, or lumbar spine in saggital and frontal planes; noted differences from AS include less pain, less limitation in movement and less symmetry. The International Spondyloarthritis Interrater Reliability Exercise (INSPIRE) has shown assessments of spinal disease in AS are also reliable in axial PsA. ${ }^{24}$

- Radiological criteria (eg, plain $x$ ray (unilateral sacroiliitis grade 2 or more), syndesmophytes, MRI changes in sacroiliac joints of bone marrow oedema, erosions, joint space narrowing). Criteria modified from AS based on data from Helliwell et al. ${ }^{25}$

Based on experience in AS, disease activity in the spine can be reliably measured by the BASDAI, where active disease has been defined by a BASDAI score $\geqslant 4$. The BASDAI can be used to measure disease activity over time; assessment should take place after 6 weeks of treatment. A treatment response, based on definition of response in AS, is defined as a BASDAI score $<3$ or a reduction by 2 points.

\section{Treatment}

See table 2 for treatment recommendations based on evidence derived in AS. Traditional oral DMARDs such as methotrexate, leflunomide and sulfasalazine, have not been shown to be effective in axial manifestations of $\mathrm{AS}^{26}$ and by extrapolation, they are not considered to be of adequate efficacy for PsA axial disease, until further data are available. 
Table 2 Treatment recommendations

\begin{tabular}{lllll}
\hline & Disease status & Treatment recommendation & Level of evidence $^{*}$ & Level of agreement $\dagger$ \\
\hline Peripheral arthritis & Mild & NSAIDs & A & $90.9 \%$ \\
& NA & $\begin{array}{l}\text { Intra-articular glucocorticoid } \\
\text { injections }\end{array}$ & D &
\end{tabular}

Comments

For control of joint but not skin symptoms May be given judiciously to treat persistently inflamed joints, if care is taken to avoid injection through psoriatic plaques. Injections to any one joint should be repeated with caution according to clinical judgment

Moderate or severe DMARDs (specific recommendations follow):

$\begin{array}{ll}\text { Sulfasalazine } & \text { A } \\ \text { Leflunomide } & \text { A } \\ \text { Methotrexate } & \text { B } \\ \text { Ciclosporine } & \text { B } \\ \text { TNF inhibitors } & \text { A }\end{array}$

Moderate or severe TNF inhibitors
Methotrexate

Fumaric acid esters

TNF inhibitors

Efalizumab

Ciclosporine

Acitretin

Alefacept

Sulfasalazine

Hydroxyurea

Leflunomide

Mycophenolate mofetil

Thioguanine

Nail disease

NA

Spinal disease

Mild to moderate

Retinoids

Oral PUVA

Ciclosporine

TNF inhibitors

NSAIDs

Physiotherapy

Education, analgesia and injection $\mathrm{A}$ of sacroiliac joint

Moderate to severe TNF inhibitors

NSAIDS, physical therapy, corticosteroids

Enthesitis

DMARDs

Moderate

TNF inhibitors

For all patients with severe or moderate peripheral arthritis. Consider for mild disease if patients do not respond to NSAIDS or intraarticular steroids. No evidence supporting DMARDs ahead of TNF inhibitors, although the effect size for TNF inhibitors is much larger than that for traditional DMARDs

For patients who fail to respond to at least one DMARD therapy. The three currently available TNF inhibitors (etanercept, infliximab and adalimumab) are equally effective for the treatment of peripheral arthritis and for the inhibition of radiographic progression. Patients with poor prognosis could be considered for TNF inhibitors even if they have not failed a standard DMARD

First-line therapies:

Phototherapy includes UVB/nbUVB, oral PUVA, bath PUVA, with or without acitretin. An initial trial of phototherapy should be made, unless it is not appropriate or if psoriasis is in areas that preclude phototherapy (ie, scalp, groin, axilla). Al forms of phototherapy are considered as a group, although many consider that PUVA therapy carries increased risk of skin cancer compared with other UV modalities. Aggressive immunosuppression should not follow extensive phototherapy (especially PUVA), given the increased risk of melanoma and non-melanoma skin cancer in this scenario

TNF inhibitors include etanercept, adalimumab and infliximab

Ciclosporine should be limited to less than 12 consecutive months because cumulative toxicity (ie, multiple courses) is not well studied Second-line therapies

Third-line therapies

TNF inhibitors include infliximab and alefacept For patients who fail therapies for mild to moderate disease

Infliximab, etanercept and adalimumab have all demonstrated efficacy in AS; the consensus was that similar treatment responses reported in AS were also likely to be observed in axial PsA

Evidence has been demonstrated for infliximab or for etanercept (in spondyloarthropathies) 
Table 2 Continued

\begin{tabular}{|c|c|c|c|c|c|}
\hline & Disease status & Treatment recommendation & Level of evidence* & Level of agreement $\uparrow$ & Comments \\
\hline \multirow[t]{4}{*}{ Dactylitis } & NA & NSAIDs & $\mathrm{D}$ & $90.2 \%$ & Usually employed initially \\
\hline & NA & Corticosteroids & $\mathrm{D}$ & & $\begin{array}{l}\text { Many clinicians rapidly progress to injected } \\
\text { steroids }\end{array}$ \\
\hline & Resistant & DMARDs & $\mathrm{D}$ & & $\begin{array}{l}\text { Nearly always in the context of co-existing active } \\
\text { disease }\end{array}$ \\
\hline & NA & Infliximab & $A$ & & Some evidence available \\
\hline
\end{tabular}

${ }^{*}$ See Methods section of manuscript for description of categories and levels of evidence.

†Percentage of survey responders who agreed or strongly agreed (see supplementary material).

AS, ankylosing spondylitis; DMARD, disease-modifying antirheumatic drug; NA, not applicable or not specifically defined; NSAID, non-steroidal anti-inflammatory drug; PsA, psoriatic arthritis; PUVA, psoralen-ultraviolet light; TNF, tumour necrosis factor; UVB, ultraviolet B light.

\section{Enthesitis}

\section{Diagnosis and assessment}

The diagnosis of enthesitis is challenging; currently three approaches have been described: clinical examination, including pain/tenderness/swelling at tendon, ligament or capsule insertion site by palpation and pressure; ultrasound with power Doppler; and MRI.

Several instruments proposed for clinical assessment of enthesitis have been tested in the INSPIRE study and were reliable in AS and PsA, but no single instrument has gained widespread acceptance. ${ }^{24}$ Another assessment modality is the visual analogue pain scale.

\section{Treatment}

See table 2 for treatment recommendations for mild, moderate, or severe enthesitis.

\section{Skin and nails}

\section{Diagnosis and assessment (for selection of topical vs systemic} therapy)

For patients with mild psoriasis, candidates for topical therapy alone must meet all of the following criteria: generally asymptomatic; minimal impact on QoL; amenable and responsive to localised therapy; less than $5 \%$ for plaque psoriasis; and no incapacity and/or disability. For patients with moderate to severe psoriasis, candidates for an addition or change to systemic and/or phototherapy should meet at least one of the following criteria: symptomatic (ie, pain, bleeding, itching); more than minimal impact on QoL; inadequate response to localised therapy; body surface area generally greater than $5 \%$ for plaque psoriasis; patients with guttate, erythrodermic, or pustular psoriasis; psoriasis in vulnerable areas (face, genitals, hands/feet, nails, scalp, or intertriginous areas); or varying degrees of incapacity and disability from psoriasis.

\section{Treatment}

See table 2 for a list of first-line, second-line and third-line systemic therapies for treatment of psoriasis.

It is important to note that the clinician should consider all agents in a treatment level before proceeding to "lower" level. Situations rendering a specific therapy as "not appropriate" include lack of response, adverse events and poor access to therapy.

Unusual clinical subsets of psoriasis can co-occur with arthritis; thus, treatment may vary from that used in psoriasis vulgaris. For erythrodermic/generalised pustular psoriasis, consider acitretin as first-line therapy, although more research is needed. For palmoplantar pustuolosis, acitretin and oral PUVA appear to result in improvement (no evidence for either one being superior), with combination of the two providing superior response. Ciclosporine and tetracycline appear to be of modest benefit. No strict recommendations can be given, due to lack of definition of treatment response, lack of controlled studies in this area. ${ }^{27}$ For treatment of hand/foot psoriasis, consider topical PUVA, soriatane, efalizumab as preferable first-line agents although more research is needed in this area.

All three TNF inhibitors have shown efficacy in phase 3 randomised controlled trials, but no head-to-head trials have been published to directly compare efficacy and safety. Some data, however, suggest that etanercept may not be as effective in patients with high BMIs. ${ }^{15}$ In psoriasis studies, etanercept efficacy was dose-dependent, with doses as high as $100 \mathrm{mg}$ per week (double the typical dose for RA and PsA patients) providing the most benefit. ${ }^{28}$

The efficacy of therapies for psoriatic nail disease is not well studied; see table 2 for existing evidence. ${ }^{29}$ Specific recommendations cannot be made due to size of studies and lack of appropriate controls.

At this time, no recommendation can be given regarding efficacy and side effect profiles of systemic corticosteroids because clinical trial data are not available. In general, monotherapy with systemic corticosteroids is to be avoided in psoriasis because skin disease can flare during or after taper. Further studies, however, are needed to evaluate the role of short-term corticosteroids in severe, pustular and erythrodermic psoriasis.

\section{Dactylitis}

Diagnosis and assessment

Dactylitis, defined as uniform swelling of a digit, is due to synovitis, tenosynovitis and enthesitis together with soft-tissue oedema. Dactylitis occurs in 16-48\% of cases of PsA, and acute dactylitis has been shown to be a clinical indicator of disease severity in PsA. ${ }^{30}$ Conversely, chronic, non-tender diffuse dactylitic swelling may be less clinically significant, although MRI appearances differ only quantitatively from acute dactylitis. Recurrent isolated dactylitis, often in the same digit(s), may be the only clinical manifestation of PsA.

\section{Treatment}

The treatment of dactylitis is largely empirical. See table 2 for treatment recommendations of initial and more resistant cases.

\section{Severity assessment in PsA}

Patients may be roughly stratified in categories of "mild", "moderate", or "severe" for peripheral arthritis, skin disease, spinal disease, enthesitis and dactylitis according to presence of criteria noted in table 3 . This table is designed to be used as a tool to assist in decision making, and rigorous adherence to the proposed stratification is not appropriate. Until numeric 
Table 3 Disease severity

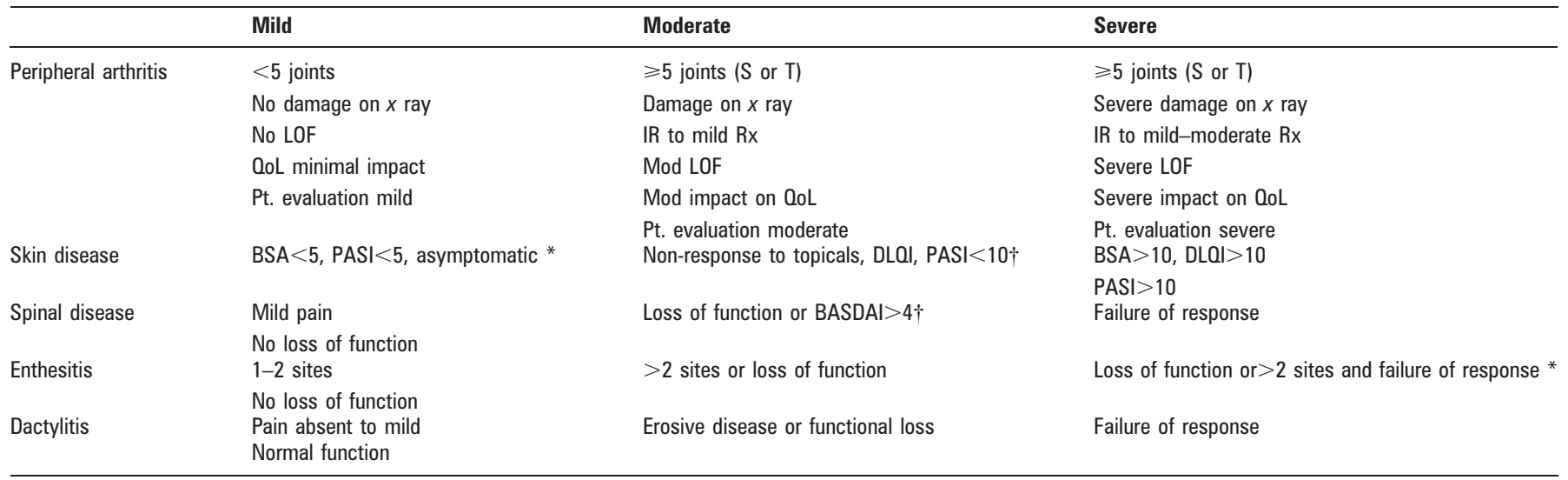

\footnotetext{
${ }^{*}$ See case 1 in table 4 ; †see case 2 in table 4.
}

S, swollen; T, tender; LOF, loss of physical function; IR, inadequate response; BSA, body surface area; BASDAI, Bath Ankylosing Spondylitis Disability Activity Index; PASI, Psoriasis Activity Severity Score; QoL, quality of life; DLOI, Dermatology Life Quality Index.

thresholds for mild, moderate and severe for the various instruments are validated, doctor judgment is required to appropriately stratify individual patients. Some patients may have multiple manifestations, and treatment decisions may be determined by the most severe clinical presentation. The synergistic impact of multiple simultaneous manifestations may be assessed with the patient global assessment, HAO and disease-specific instruments (DLOI, PsAQoL). Two case descriptions are provided in table 4.

\section{DISCUSSION}

The array of disease manifestations coupled with the wide range in disease severity and course observed in PsA present formidable challenges to the treating clinician. Therapeutic decisions must be based on thorough assessments of the different areas of involvement including the skin and nails. Of note, the cumulative negative impact of widespread inflammation at various sites can be multiplicative, leading to profound impairment of patient quality of life and function. Further complicating treatment decisions are the paucity of adequately powered placebo-controlled clinical trial data for some of the most common agents used in the treatment of PsA, most notably methotrexate. Certainly, the development of improved trial design that incorporates relevant and measurable outcomes favours biological agents since many of the older studies conducted on DMARDs are under-powered and suffer design flaws. Lastly, management of PsA often requires input from rheumatology and dermatology: any treatment recommendations must be developed based on input from groups of doctors as well as their patients. ${ }^{31} 32$

The GRAPPA organisation was founded with the mission to improve the care of PsA patients based on input from all doctors and health professionals who care for PsA patients. ${ }^{1}$ The GRAPPA Treatment Recommendations Committee received expert opinion from over 16 dermatologists; indeed, the psoriasis treatment section of this paper was developed entirely by these doctors. Of note, however, these dermatologists focused on the treatment of psoriasis in the setting of PsA; therefore, their recommendations should not be extrapolated to psoriasis alone. The section on psoriasis treatment was also broadened to include some unusual subsets that can be particularly vexing for the treating doctor with regard to diagnosis and management.

The therapeutic strategies outlined in this manuscript represent the first treatment recommendations based on a thorough review of the literature, followed by a consensus exercise among international dermatology and rheumatology experts who care for PsA patients. Input from PsA patients provided the doctors with a deeper understanding and appreciation of how treatment options and decisions are viewed

Table 4 Case descriptions

\begin{tabular}{|c|c|c|}
\hline Case & History/symptoms & Recommendation(s) \\
\hline 1 & $\begin{array}{l}\text { 19-year-old male student: } \\
\text { History of psoriasis. } \\
\text { Presented with disabling bilateral Achilles tendonitis and right plantar fasciitis. } \\
\text { Unable to bear weight. } \\
\text { Initial treatment (without sustained relief) included two different NSAIDs, a 10-day course of oral } \\
\text { corticosteroids, physiotherapy and plantar fascia injection. } \\
\text { Symptoms have been present for } 10 \text { weeks. } \\
\text { Mild scalp psoriasis that is well controlled with topical agents. }\end{array}$ & $\begin{array}{l}\text { This patient has severe enthesitis and mild skin disease (see } \\
\text { table 3), and he has failed therapies for mild and moderate } \\
\text { enthesitis; a TNF inhibitor should be considered. }\end{array}$ \\
\hline 2 & $\begin{array}{l}\text { 34-year-old male: } \\
\text { Moderate to severe psoriasis since childhood. } \\
\text { 2-year history of inflammatory back pain with unilateral grade } 2 \text { sacroiliitis on a plain film of the AP } \\
\text { pelvis; his BASDAI is } 5.6 \text {. } \\
\text { Used topical agents and phototherapy for psoriasis; has been treated with two different NSAIDS and } \\
\text { an exercise program with no change in the BASDAl. } \\
\text { No loss of function but mild impairment in QoL. } \\
\text { Percentage of BSA with plaque is } 5 \% \text {, which is having a significant negative impact on } 0 \mathrm{oL} \text {, more } \\
\text { than the back pain. } \\
\text { DLOl is } 7.2 \text {. }\end{array}$ & $\begin{array}{l}\text { This patient has moderate axial disease and moderate skin } \\
\text { involvement (see table } 3 \text { ). For his axial disease, it is } \\
\text { recommended that he have education, analgesia and } \\
\text { sacroiliac injection. For his skin disease, a systemic agent is } \\
\text { warranted. If the combination of axial and skin disease is } \\
\text { severely impairing } \mathrm{OoL} \text { and/or function, a TNF inhibitor may } \\
\text { be considered }\end{array}$ \\
\hline
\end{tabular}


by individuals who suffer from this disease. The weaknesses of these recommendations centre primarily on the lack of studies with high levels of evidence. It should be noted that many of the agents mentioned in these guidelines are not necessarily approved by appropriate regulatory agencies for these indications; for example, fumarates are neither US Federal Drug Authority (FDA) nor European Medicines Agency (EMEA) approved (they are approved only in Germany) for the therapy of plaque psoriasis, but there is sufficient high-grade evidence to warrant their inclusion as a first-line therapy. In addition, while the sample size of participating GRAPPA members seems robust, this number represents less than half of the registered members in the organisation. Although patient-reported outcomes (physical function, OoL and fatigue) have been measured and have shown positive results, particularly with TNF inhibitors, they were not the focus of our guidelines, except as part of composite scores (eg, the HAO and patient global assessment in the ACR scoring system). Finally, considerable differences persist among the members regarding how to assess severity for the various manifestations of PsA. While the group did have over $80 \%$ agreement regarding the content of the disease grid (table 3), it was with the understanding that this tool represents a starting point that will be modified as new trial data are published. The core domains and instruments for use in clinical trials and in the care of PsA patients have been identified by GRAPPA, and preliminary validation was obtained through the OMERACT process. ${ }^{33-35}$

Assessment and treatment of axial manifestations of PsA is very challenging because of the paucity of data. By consensus, it was agreed that ASAS guidelines be used. ${ }^{36}$ The cut-off point for the definition of moderate to severe disease activity in AS was chosen by ASAS to be a BASDAI score $\geqslant 4$. For BASDAI response criteria, a $50 \%$ relative change or absolute change of 2 (0-10) with expert opinion of significant improvement was chosen. However, this cut-off was formally validated by Cohen et $a l^{37}$ and Pavy et $a^{138}$ and represents an appropriate criterion to borrow from AS for axial disease in PsA until further testing can be performed prospectively in clinical trials.

Recent studies have shown that dermatologists and rheumatologists can assess skin and joint disease with a surprising degree of agreement and accuracy. ${ }^{39}$ Similar studies have been published for axial disease. ${ }^{24}$ Efforts to develop instruments for assessment of dactylitis and enthesitis are underway, and it is anticipated that these will be tested for validity in the near future. $^{24}{ }^{40}$ Ultimately, a composite assessment tool that can be applied in the office setting will allow clinicians to formulate more informed treatment decisions for individual patients.

Interest in PsA has greatly intensified over the past several years due to several factors including a better understanding of disease mechanisms, improved clinical trial design and perhaps most importantly, the arrival of effective and relatively safe biological agents that have dramatically altered the treatment paradigm. Currently, a host of new treatments are in the pipeline, many of which will offer new and possibly less expensive therapeutic options. It is anticipated that the treatment recommendations outlined in this study will be refined and serve as a template for the development of revised PsA treatment updates as new data are released.

Acknowledgements: The authors would like to thank the patients and members of GRAPPA for their valuable time and input toward drafting these treatment recommendations, Stacie Byars for her skilful coordination of the efforts for this project and Linda Melvin for assistance in manuscript preparation.

Competing interests: None declared.

\section{REFERENCES}

1. Kavanaugh AF, Ritchlin CT. Systematic review of treatments for psoriatic arthritis: an evidence based approach and basis for treatment guidelines. J Rheumatol 2006;33:1417-21.

2. Griffiths CE, Christophers E, Barker JN, Chalmers RJ, Chimenti S, Krueger GG, et al. A classification of psoriasis vulgaris according to phenotype. $\mathrm{Br} \mathrm{J}$ Dermatol 2007;156:258-62

3. McCarey D, Mclnnes IB. Psoriatic arthritis: current topics. Curr Rheumatol Rep 2007:9:442-8.

4. Setty AR, Choi HK. Psoriatic arthritis epidemiology. Curr Rheumatol Rep 2007;9:449-54.

5. Mallbris L, Ritchlin CT, Stahle M. Metabolic disorders in patients with psoriasis and psoriatic arthritis. Curr Rheumatol Rep 2006;8:355-63.

6. Peters MJ, van der Horst-Bruinsma IE, Dijkmans BA, Nurmohamed MT. Cardiovascular risk profile of patients with spondylarthropathies, particularly ankylosing spondylitis and psoriatic arthritis. Semin Arthritis Rheum 2004;34:585-92

7. Gelfand JM, Neimann AL, Shin DB, Wang X, Margolis DJ, Troxel AB. Risk of myocardial infarction in patients with psoriasis. JAMA 2006;296:1735-41.

8. Ritchlin C. Psoriatic disease - from skin to bone. Nat Clin Pract Rheumatol 2007:3:698-706

9. Gladman DD, Mease PJ. Towards international guidelines for the management of psoriatic arthritis. J Rheumatol 2006;33:1228-30.

10. Soriano ER, McHugh NJ. Therapies for peripheral joint disease in psoriatic arthritis A systematic review. J Rheumatol 2006;33:1422-30.

11. Nash P. Therapies for axial disease in psoriatic arthritis. A systematic review. J Rheumatol 2006;33:1431-4.

12. Ritchlin CT. Therapies for psoriatic enthesopathy. A systematic review. J Rheumatol 2006;33:1435-8.

13. Helliwell PS. Therapies for dactylitis in psoriatic arthritis. A systematic review. J Rheumatol 2006;33:1439-41.

14. Cassell S, Kavanaugh AF. Therapies for psoriatic nail disease. A systematic review. $J$ Rheumatol 2006:33:1452-6.

15. Boehncke WH, Prinz J, Gottlieb AB. Biologic therapies for psoriasis. A systematic review. J Rheumatol 2006;33:1447-51.

16. Strober BE, Siu K, Menon K. Conventional systemic agents for psoriasis. A systematic review. J Rheumatol 2006;33:1442-6.

17. Cook DJ, Mulrow CD, Haynes RB. Systematic reviews: synthesis of best evidence for clinical decisions. Ann Intern Med 1997:126:376-80.

18. Thalheimer W, Cook S. How to calculate effect sizes from published research articles: a simplified methodology. http://www.work-learning.com/effect sizes.htm (accessed 5 May 2008)

19. Taylor W, Gladman D, Helliwell P, Marchesoni A, Mease P, Mielants H. Classification criteria for psoriatic arthritis: development of new criteria from a large international study. Arthritis Rheum 2006;54:2665-73.

20. Gladman DD, Mease PJ, Strand V, Healy P, Helliwell PS, Fitzgerald O, et al. Consensus on a core set of domains for psoriatic arthritis. J Rheumatol 2007;34:1167-70

21. Bond SJ, Farewell VT, Schentag CT, Gladman DD. Predictors for radiological damage in psoriatic arthritis: results from a single centre. Ann Rheum Dis 2007;66:370-6.

22. Fransen J, Antoni C, Mease PJ, Uter W, Kavanaugh A, Kalden JR, et al. Performance of response criteria for assessing peripheral arthritis in patients with psoriatic arthritis: analysis of data from randomised controlled trials of two tumour necrosis factor inhibitors. Ann Rheum Dis 2006;65:1373-8.

23. Leeb BF, Andel I, Sautner J, Fassl C, Nothnagl T, Rintelen B. The Disease Activity Score in 28 joints in rheumatoid arthritis and psoriatic arthritis patients. Arthritis Rheum 2007:57:256-60.

24. Gladman DD, Inman RD, Cook RJ, Maksymowych WP, Braun J, Davis JC, et al. International spondyloarthritis interobserver reliability exercise - the INSPIRE study: ॥. Assessment of peripheral joints, enthesitis, and dactylitis. J Rheumatol 2007;34:1740-5

25. Helliwell PS, Hickling P, Wright V. Do the radiological changes of classic ankylosing spondylitis differ from the changes found in the spondylitis associated with inflammatory bowel disease, psoriasis, and reactive arthritis? Ann Rheum Dis 1998:57:135-40.

26. Zochling J, van der Heijde D, Burgos-Vargas R, Collantes E, Davis JC Jr, Dijkmans B, et al. ASAS/EULAR recommendations for the management of ankylosing spondylitis. Ann Rheum Dis 2006:65:442-52.

27. Marsland AM, Chalmers RJG, Hollis S, Leonardi-Bee J, Griffiths CEM. Interventions for chronic palmoplantar pustulosis. Cochrane Database Syst Rev 2006;1:CD001433.

28. Leonardi CL, Powers JL, Matheson RT, Goffe BS, Zitnik R, Wang A, et al. Etanercept as monotherapy in patients with psoriasis. N Engl J Med 2003;349:2014-22.

29. Rich P, Griffiths CE, Reich K, Nestle FO, Scher RK, Li S, et al. Baseline nail disease in patients with moderate to severe psoriasis and response to treatment with infliximab during 1 year. J Am Acad Dermatol 2008:58:224-31.

30. Brockbank JE, Stein M, Schentag CT, Gladman DD. Dactylitis in psoriatic arthritis: a marker for disease severity? Ann Rheum Dis 2005;64:188-90.

31. Gordon KB, Ruderman EM. The treatment of psoriasis and psoriatic arthritis: an interdisciplinary approach. J Am Acad Dermatol 2006:54(Suppl 2):S85-91.

32. Mease P. Management of psoriatic arthritis: the therapeutic interface between rheumatology and dermatology. Curr Rheumatol Rep 2006:8:348-54.

33. Gladman DD, Helliwell P, Mease PJ, Nash P, Ritchlin C, Taylor W. Assessment of patients with psoriatic arthritis: a review of currently available measures. Arthritis Rheum 2004:50:24-35. 
34. Gladman DD, Mease PJ, Healy P, Helliwell PS, Fitzgerald O, Cauli A, et al. Outcome measures in psoriatic arthritis. J Rheumatol 2007;34:1159-66.

35. Mease PJ, Antoni CE, Gladman DD, Taylor WJ. Psoriatic arthritis assessment tools in clinical trials. Ann Rheum Dis. 2005:64(Suppl 2):ii49-54.

36. Braun J, Pham T, Sieper J, Davis J, van der Linden S, Dougados M, et al. International ASAS consensus statement for the use of anti-tumour necrosis factor agents in patients with ankylosing spondylitis. Ann Rheum Dis 2003;62:817-24.

37. Cohen JD, Cunin P, Farrenq V, Oniankitan O, Carton L, Chevalier X, et al. Estimation of the Bath Ankylosing Spondylitis Disease Activity Index cutoff for perceived symptom relief in patients with spondyloarthropathies. J Rheumatol 2006;33:79-81.
38. Pavy S, Brophy S, Calin A. Establishment of the minimum clinically important difference for the bath ankylosing spondylitis indices: a prospective study. $J$ Rheumatol 2005;32:80-5.

39. Chandran V, Cook R, Helliwell P, Kavanaugh A, McHugh N, Mease P, et al. International Multi-centre Psoriasis and psoriatic Arthritis Reliability Trial (GRAPPAIMPART): Assessment of skin, joints, nails and dactylitis. Arthritis Rheum 2007:56(Suppl 9):S798.

40. Helliwell PS, Firth J, Ibrahim GH, Melsom RD, Shah I, Turner DE. Development of an assessment tool for dactylitis in patients with psoriatic arthritis. $J$ Rheumatol 2005; 32:1745-50.

\section{Keep up to date: sign up for our alerting services}

Find out automatically when an article is published on a specific topic or by a particular author. We can also alert you when an article is cited or if an eLetter or correction is published. You can also choose to be alerted when a new issue is published online [and when we post articles Online First]. Check out the New Content Alerts and Citation tracker from the Online tools section on the home page. 